ARTICLE

Received 4 Nov 2015 | Accepted 13 May 2016 | Published 21 Jun 2016

DOI: $10.1038 /$ ncomms11932

OPEN

\title{
Activation of STING requires palmitoylation at the Golgi
}

Kojiro Mukai ${ }^{1}, 2$, Hiroyasu Konno ${ }^{3}$, Tatsuya Akiba ${ }^{1}$, Takefumi Uemura ${ }^{4}$, Satoshi Waguri ${ }^{4}$, Toshihide Kobayashi $^{2}$, Glen N. Barber ${ }^{3}$, Hiroyuki Arai ${ }^{1,5}$ \& Tomohiko Taguchi, ${ }^{1,5}$

Stimulator of interferon genes (STING) is essential for the type I interferon response against DNA pathogens. In response to the presence of DNA and/or cyclic dinucleotides, STING translocates from the endoplasmic reticulum to perinuclear compartments. However, the role of this subcellular translocation remains poorly defined. Here we show that palmitoylation of STING at the Golgi is essential for activation of STING. Treatment with palmitoylation inhibitor 2-bromopalmitate (2-BP) suppresses palmitoylation of STING and abolishes the type I interferon response. Mutation of two membrane-proximal Cys residues (Cys88/91) suppresses palmitoylation, and this STING mutant cannot induce STING-dependent host defense genes. STING variants that constitutively induce the type I interferon response were found in patients with autoimmune diseases. The response elicited by these STING variants is effectively inhibited by 2-BP or an introduction of Cys88/91Ser mutation. Our results may lead to new treatments for cytosolic DNA-triggered autoinflammatory diseases.

\footnotetext{
${ }^{1}$ Department of Health Chemistry, Graduate School of Pharmaceutical Sciences, University of Tokyo, 7-3-1, Hongo, Bunkyo-ku, Tokyo 113-0033, Japan.

${ }^{2}$ Lipid Biology Laboratory, RIKEN, 2-1, Hirosawa, Wako-shi, Saitama 351-0198, Japan. ${ }^{3}$ Department of Cell Biology and Sylvester Comprehensive Cancer Center, University of Miami School of Medicine, Miami, Florida 33136, USA. ${ }^{4}$ Department of Anatomy and Histology, Fukushima Medical University School of Medicine, Hikarigaoka, Fukushima 960-1295, Japan. ${ }^{5}$ Pathological Cell Biology Laboratory, Graduate School of Pharmaceutical Sciences, University of Tokyo, Tokyo 113-0033, Japan. Correspondence and requests for materials should be addressed to H.A. (email: harai@mol.f.u-tokyo.ac.jp)

or to T.T. (email: tom_taguchi@mol.f.u-tokyo.ac.jp).
} 
T he innate immune response is critical for efficient host defense against microbial invasion. Invading pathogens are identified by pattern recognition receptors in the host cell, which initiates a series of signalling events that leads to the production of type I interferons, proinflammatory cytokines and other downstream antiviral proteins ${ }^{1,2}$. The pattern recognition receptors include Toll-like receptors, RIG-I-like receptors and nucleotide-binding domain and leucine-rich repeat-containing receptors that sense microbial molecules such as CpG DNA, viral RNAs and lipopolysaccharides ${ }^{3-5}$. Furthermore, an endoplasmic reticulum (ER)-associated molecule referred to as stimulator of interferon genes (STING, also known as MITA, ERIS, MPYS or TMEM173 $)^{6-10}$ has recently been shown to control a new sensing pathway that is essential for detecting cytosolic DNA or cyclic dinucleotides (CDNs) which include cyclic GMP-AMP (cGAMP) $^{11-13}$.

Besides the essential roles of STING in protecting the host against DNA pathogens, STING is also involved in the pathogenesis of autoinflammation caused by self-DNA in murine models (DnaseII ${ }^{-/-}$and DnaseIII $\left.(\text {Trex1) })^{-/-}\right)^{14-16}$. This suggests that STING may also be involved in human autoinflammatory disorders such as Aicardi-Goutieres syndrome and systemic lupus erythematosus that are associated with mutations of human DNase $\mathrm{III}^{17,18}$. Furthermore, mutations in STING are found in patients with an autoinflammatory disease called STING-associated vasculopathy with onset in infancy $(\mathrm{SAVI})^{19}$ and more recently in patients with lupus-like syndromes ${ }^{20}$. The STING variants found in SAVI patients appear to be constitutively activated without CDNs and interestingly do not localize to the $\mathrm{ER}^{20,21}$.

After DNA and/or CDN binding, STING translocates from the ER to perinuclear compartments that include the Golgi, endosomes and autophagy-related compartments ${ }^{6,22}$. Treatment with brefeldin A (BFA) or expression of Shigella effector IpaJ, which blocks ER-to-Golgi traffic, abolishes the STING-dependent signalling events that include phosphorylation of TANK-binding kinase 1 (TBK1) and the transcription factor interferon regulatory factor 3 (IRF3) and induction of interferon $\beta$ (IFN $\beta)^{6,21,23}$. These results, together with the observation that SAVI-STINGs (activated without $\mathrm{CDNs}$ ) do not localize to the ER, all indicate the contribution of post-ER compartments to the activation of STING. However, the molecular mechanism underlying the activation of STING in post-ER compartments is not understood.

In the present study, we showed that STING is palmitoylated at the Golgi and this post-translational modification is required for the activation of STING-dependent downstream signalling for the type I interferon response.

\section{Results}

Post-ER trafficking route of STING after stimulation. To examine the intracellular behaviour of STING, we added an amino-terminal EGFP tag to mouse STING (EGFP-mSTING) and used DMXAA, a membrane-permeable mouse-specific STING agonist. When EGFP-mSTING was transiently expressed in HEK293T cells that lack endogenous STING ${ }^{12}$, it activated the IRF3 promoter in a DMXAA-dependent manner (Supplementary Fig. 1a), indicating that tagging EGFP to mSTING did not impair the STING activity. EGFP-mSTING was then stably expressed in COS-1 cells (emsCOS-1 cells), a cell line with distinctly separate organelles ${ }^{24}$. emsCOS-1 cells induced downstream genes of STING, such as IFNB, IL8 and TNF on DMXAA stimulation (Supplementary Fig. 1b). Following the binding of DMXAA or CDNs, STING recruits TBK1 to induce autophosphorylation of TBK1 (ref. 23). Phosphorylated TBK1 then phosphorylates transcription factor IRF3 to stimulate transcription of $I F N B^{25}$.
Phosphorylation of TBK1 peaked at $60 \mathrm{~min}$ after DMXAA stimulation in emsCOS-1 cells (Fig. 1a). Phosphorylated IRF3 was observed for $60-120 \mathrm{~min}$ (Fig. 1a). As was shown previously for endogenous STING ${ }^{23}$, EGFP-mSTING localized at the ER in unstimulated cells and exited the ER after stimulation (Fig. 1b). Co-immunostaining with GM130, a Golgi protein, showed that EGFP-mSTING mostly localized to the Golgi at $60 \mathrm{~min}$ (Fig. 1b). Phosphorylated TBK1 was partly co-localized with EGFPmSTING at this time (Fig. 1c). EGFP-mSTING co-localized with rab11, a recycling endosomal protein at $240 \mathrm{~min}$, and with p62, a lysosomal/autophagic protein at $480 \mathrm{~min}$ (Supplementary Fig. 1c). These results suggest that STING sequentially moved from the ER to the Golgi to recycling endosomes and then to the lysosomal/autophagic membranes after stimulation and that phosphorylation of TBK1, a hallmark of STING activation, occurred at the Golgi.

STING activates TBK1 at the TGN. We examined whether inhibition of membrane trafficking affected the phosphorylation of TBK1. Treatment with BFA, which blocks ER-to-Golgi traffic ${ }^{26}$ (Supplementary Fig. 2a), suppressed phosphorylation of TBK1 and IRF3 (Fig. 1d) $)^{6,23}$. Low temperature $\left(20^{\circ} \mathrm{C}\right)$ impairs membrane traffic from the Golgi, but not from the ER to the Golgi ${ }^{27}$. EGFP-mSTING still localized to the Golgi $480 \mathrm{~min}$ after DMXAA stimulation at $20^{\circ} \mathrm{C}$ (Supplementary Fig. 2b), while EGFP-mSTING localized to the lysosomal/autophagic membranes at that time at $37^{\circ} \mathrm{C}$ (Supplementary Fig. 1c). Under the low temperature condition, DMXAA-induced phosphorylation of TBK1 and IRF3 even more strongly than at $37^{\circ} \mathrm{C}$ (Fig. 1d). These data suggested that the Golgi was the organelle where STING activated TBK1 and IRF3.

The Golgi is a polarized organelle $e^{28}$ that has distinct functional domains, such as the cis-Golgi network (CGN) and trans-Golgi network (TGN). Treatment of cells with the microtubuledepolymerizing agent nocodazole results in dispersed Golgi stacks in the cytoplasm, and this fragmentation facilitates the analysis of cis-to-trans polarity of the Golgi ${ }^{29}$. With this method, we found that, while STING localized at both the CGN and TGN, phosphorylated TBK1 was confined to a subdomain of the TGN (Fig. 1e-g and Supplementary Fig. 3). We also found that phosphorylated TBK1 was confined to a subdomain of the TGN in mouse embryonic fibroblasts (MEFs) (Supplementary Fig. 4). Immunoelectron microscopy of emsCOS-1 cells $60 \mathrm{~min}$ after DMXAA stimulation showed that phosphorylated TBK1 was mostly localized to tubular or vesicular profiles adjacent to the TGN46-positive structures in the TGN region (Fig. 1h).

STING palmitoylation is required for the type I IFN response. We hypothesized that STING is post-translationally modified at the TGN to activate downstream signalling and found that palmitoylation of STING was required for the type I interferon response. Using a $\left[{ }^{3} \mathrm{H}\right]$ palmitate metabolic labelling approach, we found that $\left[{ }^{3} \mathrm{H}\right]$ labelling of STING was greatly increased after DMXAA stimulation (Fig. 2a). The timing of the labelling of STING correlated well with the timing of the phosphorylation of TBK1 in emsCOS-1 cells (Fig. 1a). STING localized to the Golgi 40-60 min after DMXAA stimulation of emsCOS-1 cells (Fig. 1b). These results suggested that palmitoylation of STING occurred at the Golgi and participated in the type I interferon response. Although STING already localized to the Golgi $20 \mathrm{~min}$ after the stimulation (Fig. 1b), at that time $\left[{ }^{3} \mathrm{H}\right]$ labelling of STING was as weak as before the stimulation, suggesting that palmitoylation of STING occurred in later Golgi compartments that include the TGN. We also found the stimulation-dependent $\left[{ }^{3} \mathrm{H}\right]$ labelling of STING in MEFs (Fig. 2b and Supplementary Fig. 5a). The timing 
of $\left[{ }^{3} \mathrm{H}\right]$ labelling of STING was correlated with that of phosphorylation of TBK1 and IRF3 (Fig. 2b) and that of the Golgi localization of STING (Supplementary Fig. 6). The palmitoylation of STING lingered even after STING was transported to the degradation compartments (for example, 240 min after DMXAA stimulation in Fig. 2b), suggesting that depalmitoylation of STING did not occur during its transport from the Golgi to the degradation compartments.
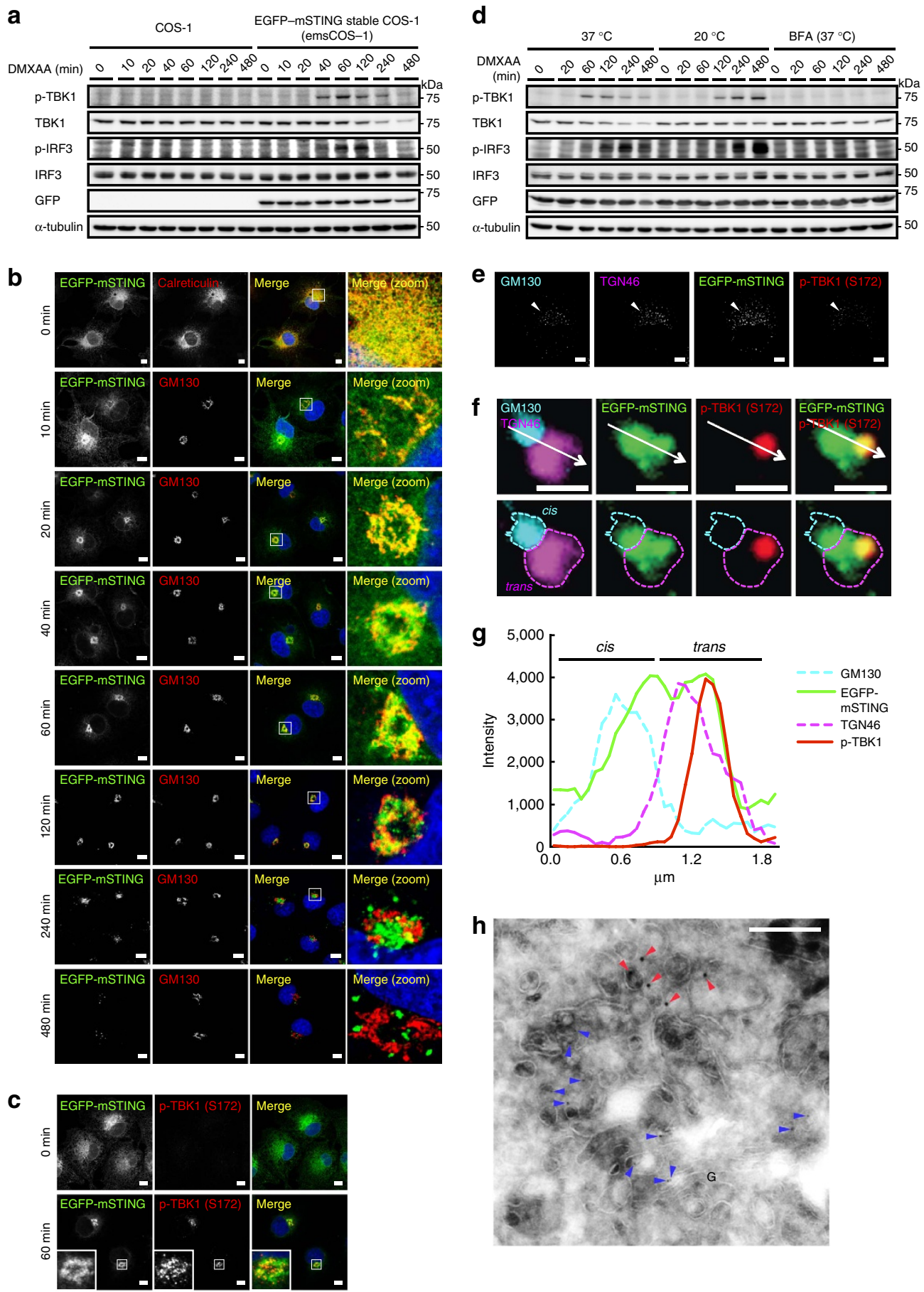

Figure 1 | STING activates TBK1 at the Golgi. (a) Western blots of cell lysates of emsCOS-1 cells stimulated with DMXAA for the indicated times. (b) Cells were stimulated with DMXAA for the indicated times, fixed, permeabilized and stained for calreticulin (an ER protein) or GM130 (a Golgi protein). Nuclei were stained with DAPI (blue). Scale bars, $10 \mu \mathrm{m}$. (c) Immunostaining of phosphorylated TBK1 after the treatment with DMXAA (0 or $1 \mathrm{~h}$ ). Scale bars, $10 \mu \mathrm{m}$. (d) The effect of low temperature $\left(20^{\circ} \mathrm{C}\right.$ ) or BFA on phosphorylation of TBK1 and IRF3. (e) Immunostaining of phosphorylated TBK1 (red) in cells treated with nocodazole and DMXAA. GM130 (a CGN protein, cyan) and TGN46 (a TGN protein, magenta) were co-stained. Scale bars, $10 \mu$ m. (f) The mini-Golgi indicated by arrow (e) was magnified. The cis and trans-regions of the mini-Golgi were outlined in the images at the bottom row. Scale bars, $1 \mu \mathrm{m}$. (g) Fluorescence intensity profile along the arrow (f) is shown. (h) emsCOS-1 cells were stimulated with DMXAA for $1 \mathrm{~h}$, fixed and processed for ultrathin-cryosections. They were immunostained with anti-p-TBK1 (rabbit) and anti-TGN46 (sheep) antibodies. As secondary antibodies, colloidal gold particle-conjugated donkey anti-rabbit antibody $(12 \mathrm{~nm})$ and donkey anti-sheep antibody $(6 \mathrm{~nm})$ were used. Red and blue arrowheads indicate p-TBK1 and TGN46 labelling, respectively. G, the Golgi stack. Scale bars, $200 \mathrm{~nm}$. 
a

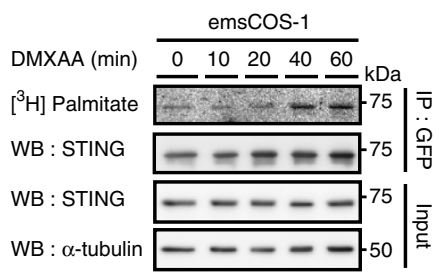

b

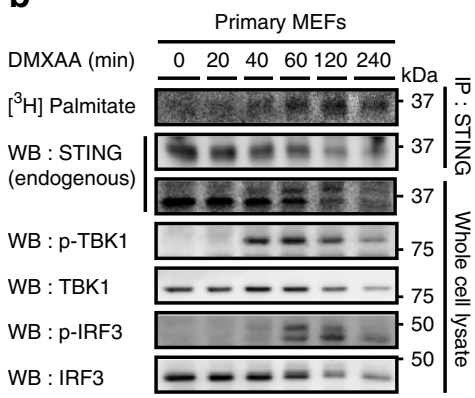

C

g

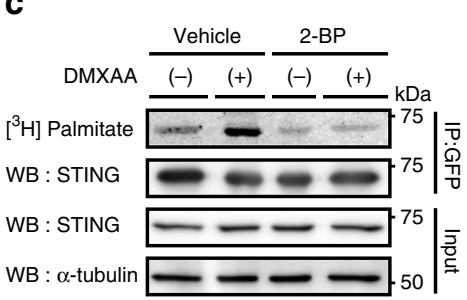

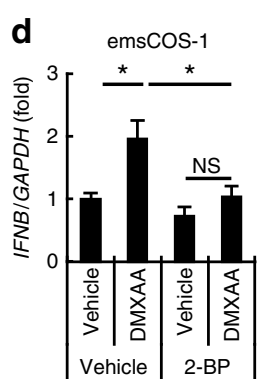

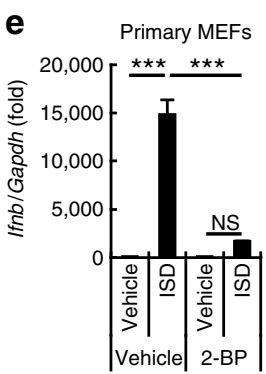

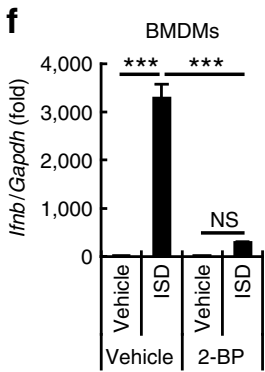

h

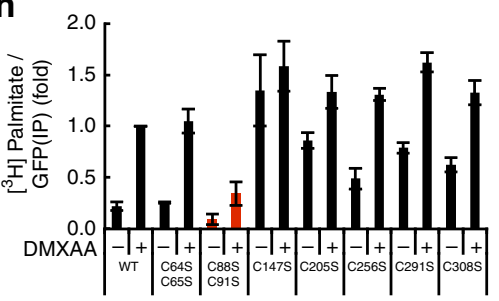

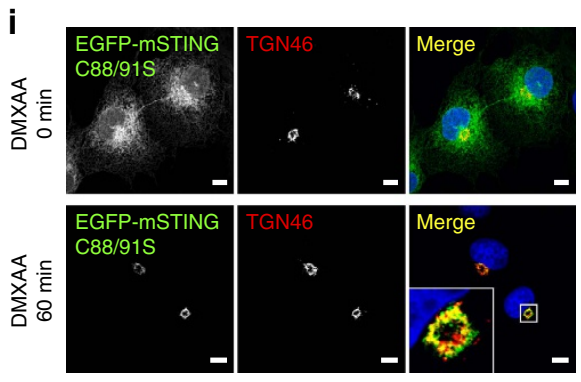
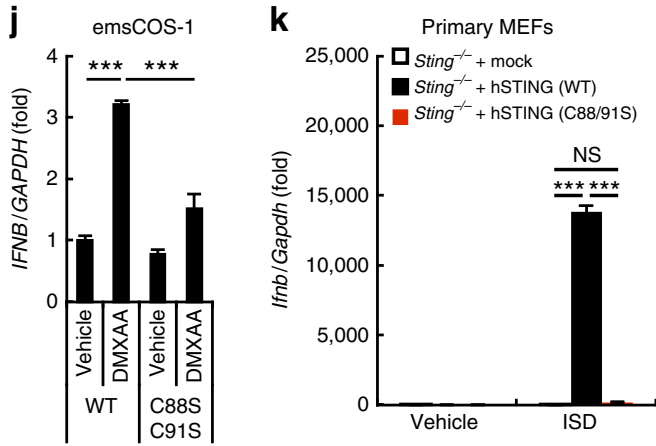

Figure 2 | Palmitoylation at Cys88/91 of STING is required for the type I interferon response. (a) emsCOS-1 cells were starved for $1 \mathrm{~h}$, followed by the incubation with $\left[{ }^{3} \mathrm{H}\right]$ palmitate for $1 \mathrm{~h}$. Cells were then stimulated with DMXAA for the indicated times. Cell lysates were prepared and EGFP-STING was immunoprecipitated with anti-GFP antibody. Cell lysates and the immunoprecipitates were analysed by western blot and autoradiography. (b) Primary MEFs were examined as in a. For immunoprecipitation, anti-STING antibody was used. (c) The effect of $50 \mu \mathrm{M} 2-\mathrm{BP}$ on the palmitoylation of STING in emsCOS-1 cells. (d) Quantitative real-time PCR ( $\mathrm{RRT}$-PCR) of the expression of IFN $\beta$ in emsCOS-1 cells that were pretreated with vehicle or $50 \mu M$ 2-BP for $1 \mathrm{~h}$ and then stimulated with DMXAA for $12 \mathrm{~h}$. (e,f) qRT-PCR of the expression of IFN $\beta$ in primary MEFs (e) or BMDMs (f) that were pretreated with vehicle or $50 \mu \mathrm{M} 2$-BP for $1 \mathrm{~h}$ and then stimulated with ISD for $3 \mathrm{~h}$. (g) COS-1 cells that stably express EGFP-mouse STING with indicated Cys mutations were metabolically labelled, stimulated with DMXAA, and analysed by western blot and autoradiography. (h) The band intensities in $\mathbf{g}$ were quantified and $\left[{ }^{3} \mathrm{H}\right.$ palmitate]/[GFP] were calculated. The data are normalized to the value of WT-STING with DMXAA treatment and represent mean \pm s.e.m. of two independent experiments. (i) COS-1 cells that stably express STING (C88/91S) were stimulated with DMXAA for $1 \mathrm{~h}$, fixed, permeabilized and stained for TGN46 (red). Nuclei were stained with DAPI (blue). Scale bars, $10 \mu \mathrm{m}$. (j) COS-1 cells that stably express STING (WT or C88/91S) were stimulated with DMXAA for $12 \mathrm{~h}$. qRT-PCR of the expression of IFN $\beta$ was then performed. (k) Primary Sting ${ }^{-/-}$MEFs were reconstituted with human STING variants using retroviruses. The cells were stimulated with ISD for $6 \mathrm{~h}$, and qRT-PCR of the expression of IFN $\beta$ was performed. Data in $\mathbf{d}, \mathbf{e}, \mathbf{f}, \mathbf{j}$ and $\mathbf{k}$ are mean \pm s.e.m. from three independent experiments. ${ }^{\star} P<0.01$, ${ }^{\star \star}{ }^{\star} P<0.001$, NS, not significant (one-way analysis of variance).

Treating emsCOS-1 cells with the palmitoylation inhibitor 2-BP effectively abolished the DMXAA-induced incorporation of $\left[{ }^{3} \mathrm{H}\right]$ into STING (Fig. 2c), confirming the palmitoylation of STING. More importantly, 2-BP suppressed DMXAA-, or interferon-stimulatory DNA (ISD)-triggered induction of downstream genes, such as IFNB, IL8, IL6 and TNF in emsCOS-1 cells, primary MEFs and bone marrow-derived macrophages (BMDMs) (Fig. 2d-f and Supplementary Fig. 7). 
a

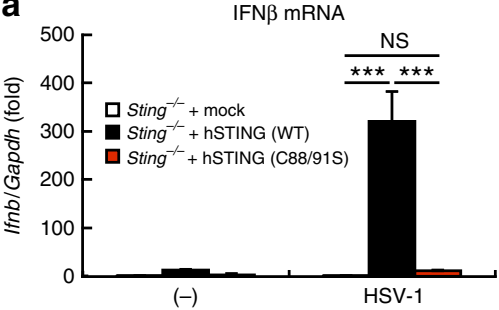

b

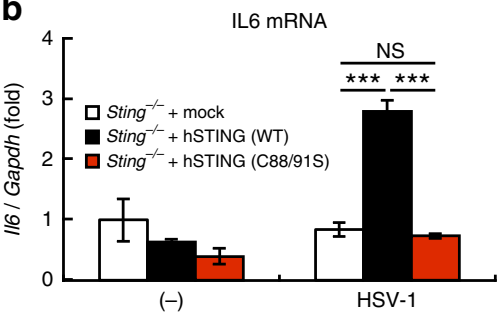

c

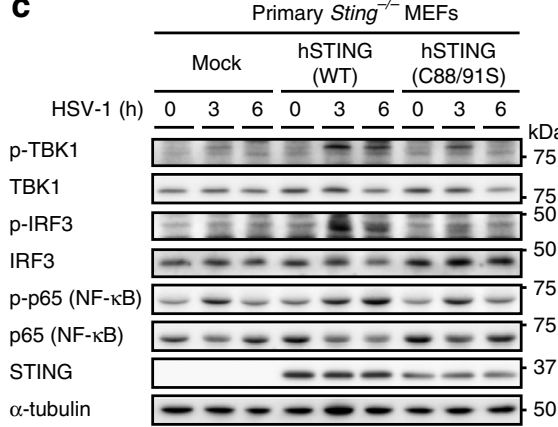

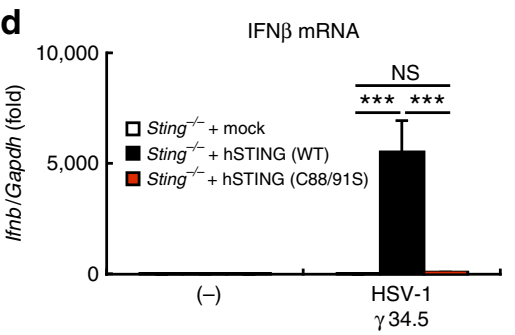

e

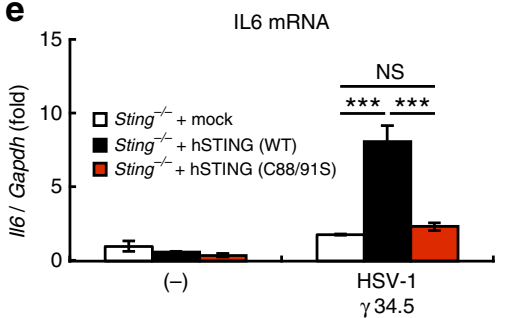

f

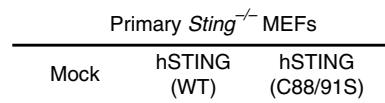

HSV-1 34.5 (h) $036 \frac{6}{03} \frac{6}{0} \frac{3}{6}$

p-TBK1

TBK1

p-IRF3

IRF3

p-p65 (NF-kB)

p65 (NF-kB)

STING

$\alpha$-tubulin

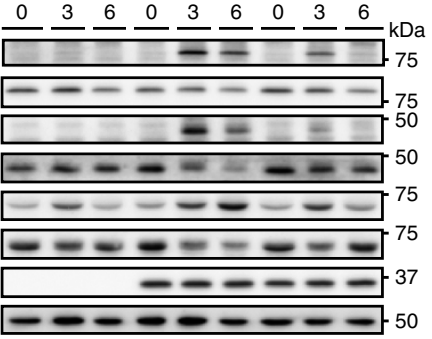

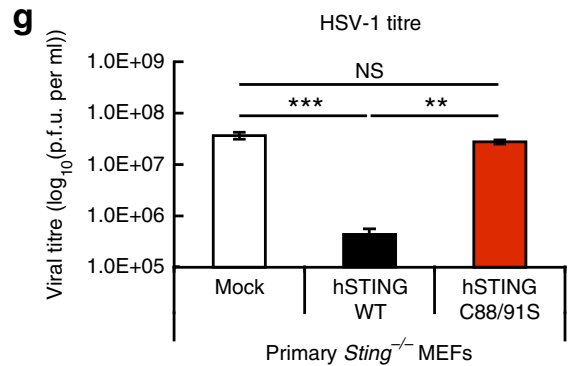

Figure 3 | STING C88/91S mutant is unable to induce the type I interferon response against HSV-1 infection. (a,b) Primary Sting ${ }^{-/-}$MEF cells were reconstituted with human STING variants using retroviruses. The cells were infected with HSV-1 (multiplicity of infection $(\mathrm{MOI})=10$ ) for $3 \mathrm{~h}$, and qRT-PCR was performed (a, IFN $\beta$ mRNA; b, IL6 mRNA). (c) Cell lysates were prepared the indicated times after HSV-1 infection, and analysed by western blot. (d-f) Cellular response to HSV-1 $\gamma 34.5$ was analysed as (a-c). (g) Reconstituted primary Sting -/- MEF cells with human STING (WT or C88/91) were infected with HSV-1 $(\mathrm{MOI}=1)$ for $24 \mathrm{~h}$, and then plaque assay was performed. Data in $\mathbf{a}, \mathbf{b}, \mathbf{d}, \mathbf{e}$ and $\mathbf{g}$ are mean \pm s.e.m. from three independent experiments. ${ }^{\star \star} P<0.005,{ }^{\star \star \star} P<0.001, N S$, not significant (one-way analysis of variance).

The effect of 2-BP was selective for STING-mediated cytosolic DNA sensing over other innate immune signalling, since we did not observe drastic effects of 2 -BP on the Toll-like receptor 3 pathway for the detection of extracellular/luminal doublestranded RNAs or on the RIG-I-like receptor (RLR) pathway for the detection of cytosolic RNAs (Supplementary Fig. 8). 2-BP did not impair the translocation of STING from the ER to the Golgi (Supplementary Fig. 9a), suggesting that palmitoylation may not be required for the binding of DMXAA to STING. 2-BP also did not impair the post-Golgi trafficking of STING from the Golgi to lysosomal/autophagic membranes (Supplementary Fig. 9a) or the degradation of STING (Supplementary Fig. 10a,b).

Cysteine (Cys) residues are the site of protein palmitoylation ${ }^{30}$. Mammalian STINGs have several conserved Cys residues that may be localized in the cytoplasmic region or near the end of the transmembrane region $^{31}$ (Supplementary Fig. 11). We generated STING mutants with single Cys to Ser substitutions (Cys147, 205, 256, 291 and 308) or dual substitutions (Cys64/65 and Cys88/91) for adjacent or proximal Cys residues, and found that the Cys88/91 dual mutation resulted in the drastic loss of palmitoylation (Fig. 2g,h). Single mutations of Cys88 or Cys91 also reduced $\left[{ }^{3} \mathrm{H}\right]$ labelling, but to a lesser extent (Supplementary Fig. 12). STING (C88/91S), like the wild type (WT), moved from the ER to the Golgi after DMXAA stimulation (Fig. 2i), but could not induce DMXAA-triggered downstream genes (Fig. $2 \mathrm{j}$ and Supplementary Fig. 13a,b). The post-Golgi trafficking and the degradation of STING (C88/91S) were essentially similar to those of STING (WT) (Supplementary Figs 9b and 10c). These data suggested that palmitoylation at Cys88/91 was critical for the type I interferon response, but not for the trafficking of STING. 
a
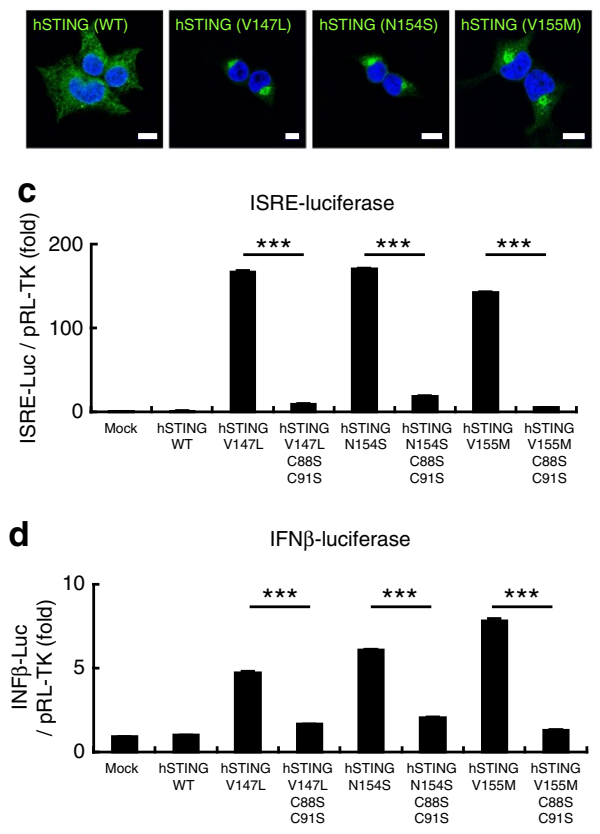

e
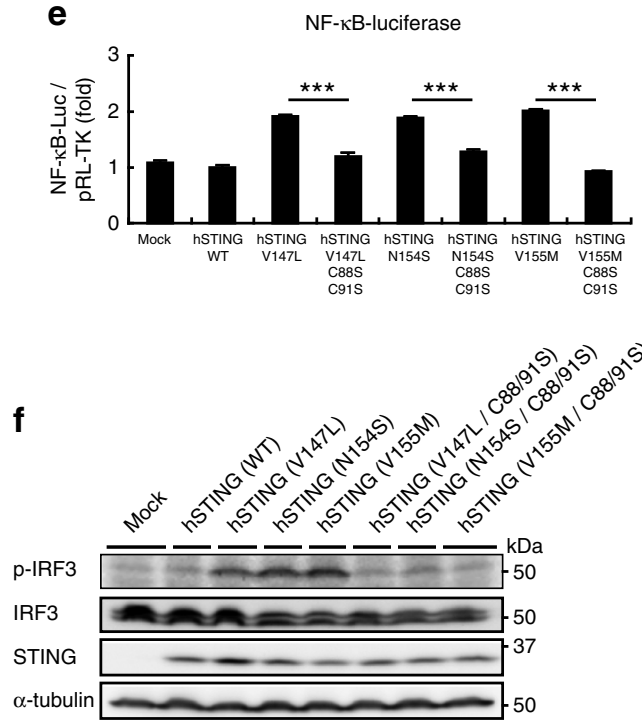

b

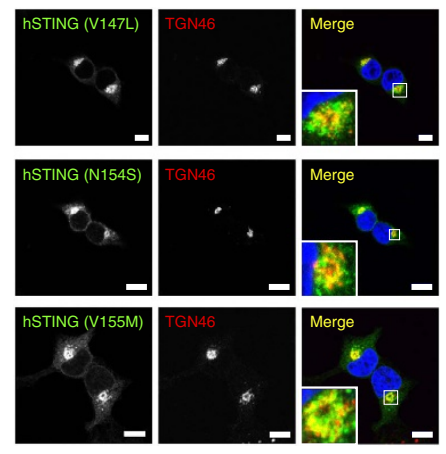

g

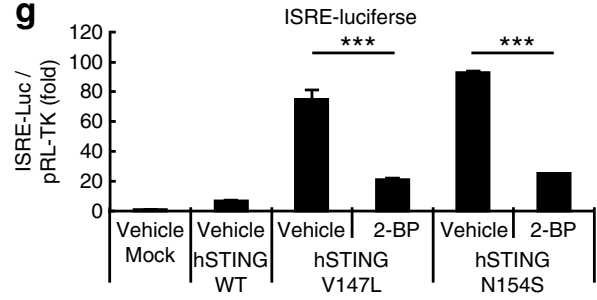

h

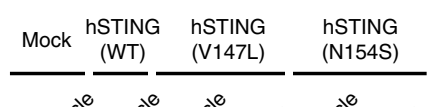

p-IRF3

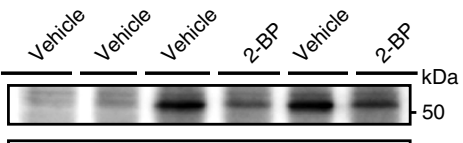

IRF3

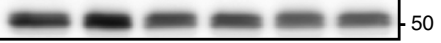

STING

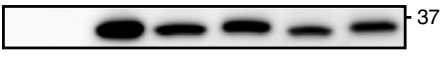

$\alpha$-tubulin

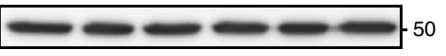

Figure 4 | Suppression of STING palmitoylation inhibits the type I interferon response elicited by the mutant STING variants associated with SAVI. (a) HEK293T cells were transfected as indicated, fixed, permeabilized and stained with anti-STING antibody. (b) Co-immunostaining of cells in a with anti-TGN46 (a TGN protein). Nuclei were stained with DAPI (blue). Scale bars, $10 \mu \mathrm{m}$. (c-e) HEK293T cells were transfected as indicated, together with an ISRE (also known as PRDIII or IRF-E)-luciferase reporter (c), IFN $\beta$-luciferase reporter (d) and NF-kB-luciferase reporter (e) for $24 \mathrm{~h}$. Luciferase activity was then measured. (f) Cells were transfected as indicated. Cell lysates were prepared $24 \mathrm{~h}$ after transfection, and analysed by western blot. (g) HEK293T cells that stably express SAVI-STING (V147L or N154S) were transfected with an ISRE-luciferase reporter for 24 h. Luciferase activity was then measured. 2-BP $(50 \mu \mathrm{M})$ was added $6 \mathrm{~h}$ after the transfection. (h) Cells that stably express SAVI-STINGs were treated with $50 \mu \mathrm{M} 2$-BP for $24 \mathrm{~h}$. Cell lysates were then prepared, and analysed by western blot. Data in $\mathbf{c}, \mathbf{d}, \mathbf{e}$ and $\mathbf{g}$ are mean \pm s.e.m. from three independent experiments. ${ }^{\star \star \star} P<0.001$ (one-way analysis of variance).

Although the Cys88/91 dual mutation resulted in the significant loss of palmitoylation, some labelling was still detected after DMXAA stimulation. STING (WT and C88/91S) was treated with hydoxylamine, a treatment that cleaves palmitoyl-thioester bonds $^{32}$ (Supplementary Fig. 5b). The treatment of stimulated STING (WT) and STING (C88/91S) mostly reduced the $\left[{ }^{3} \mathrm{H}\right]$ labelling. These results indicated that in addition to palmitoylation at Cys88/91, stimulated STING in emsCOS-1 cells undergoes palmitoylation at Cys residue (s) other than Cys88/91. In the case of STING that was reconstituted into
MEFs, the $\left[{ }^{3} \mathrm{H}\right]$ labelling of STING also diminished after the hydroxylamine treatment (Supplementary Fig. 5a). STING (C88/91S) in MEFs could not induce ISD-triggered downstream genes (Fig. $2 \mathrm{k}$ and Supplementary Fig. 13c-e), thus further supporting the role of palmitoylation at Cys88/91 of STING in the type I interferon response.

We also examined whether the C88/91S mutant activated the type I interferon response that is elicited by cGAMP, an endogenous STING ligand ${ }^{11}$. STING, when expressed at low levels in HEK293T cells, showed CDNs-dependent type I 
a

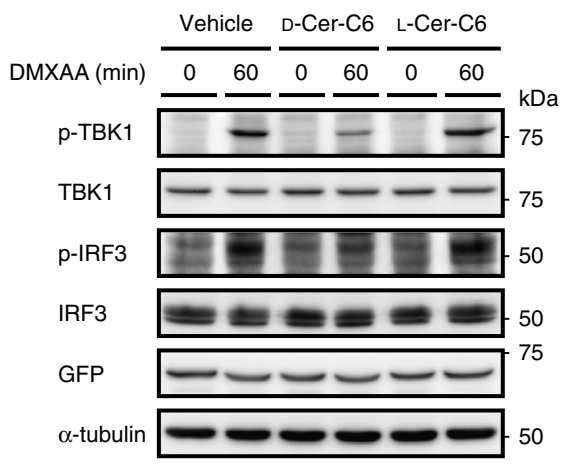

b

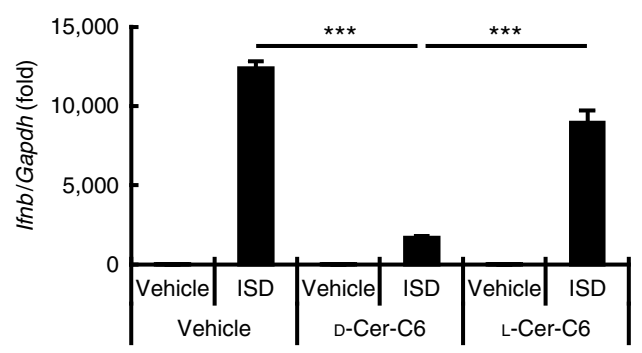

C

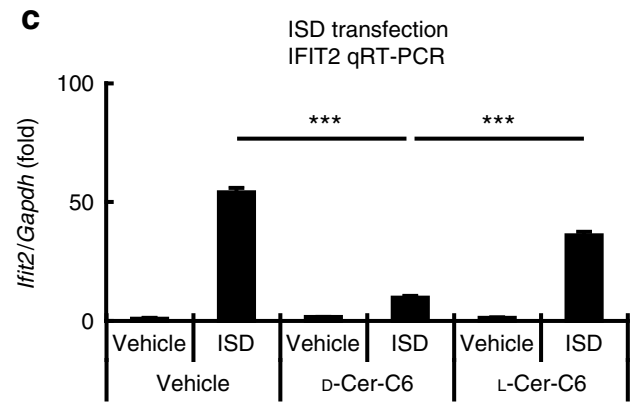

d
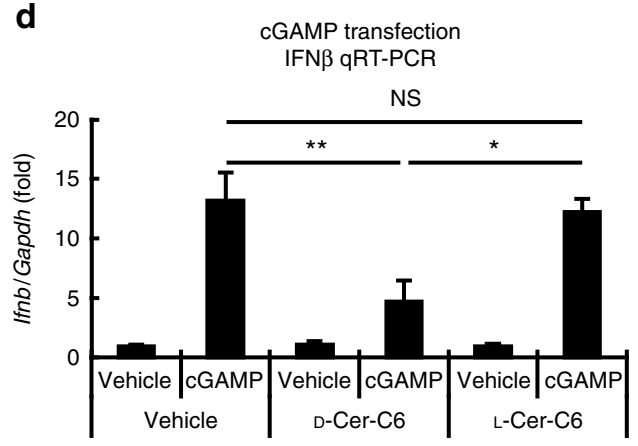

e

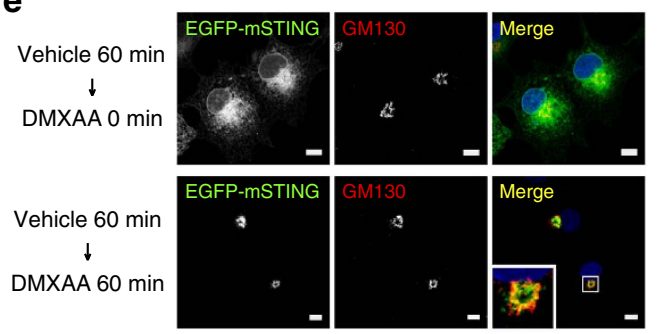

D-Cer-C6 $60 \mathrm{~min}$
$\downarrow$
DMXAA 0 min
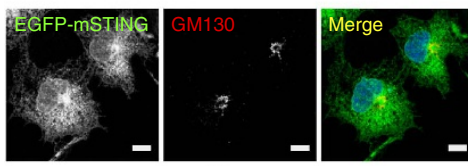

EGFP-mSTING

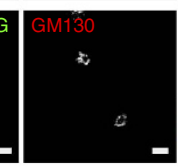

Merge

D-Cer-C6 60 min

$\downarrow$

DMXAA 60 min
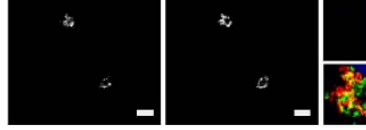

L-Cer-C6 60 min
$\downarrow$
DMXAA 0 min
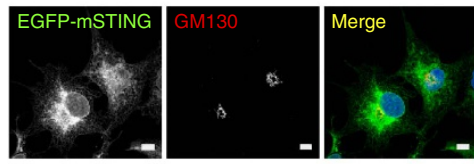

L-Cer-C6 60 min $\downarrow$
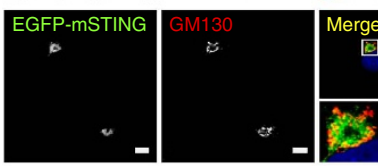

Merge

DMXAA $60 \mathrm{~min}$

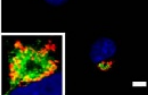

f

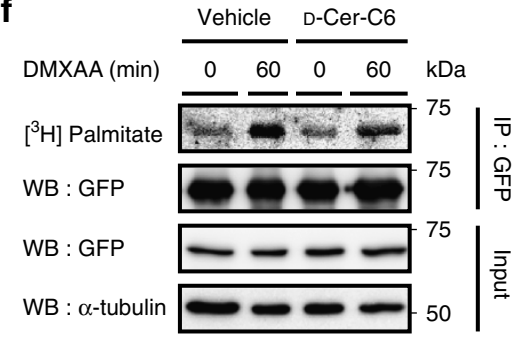

$\mathbf{g}$

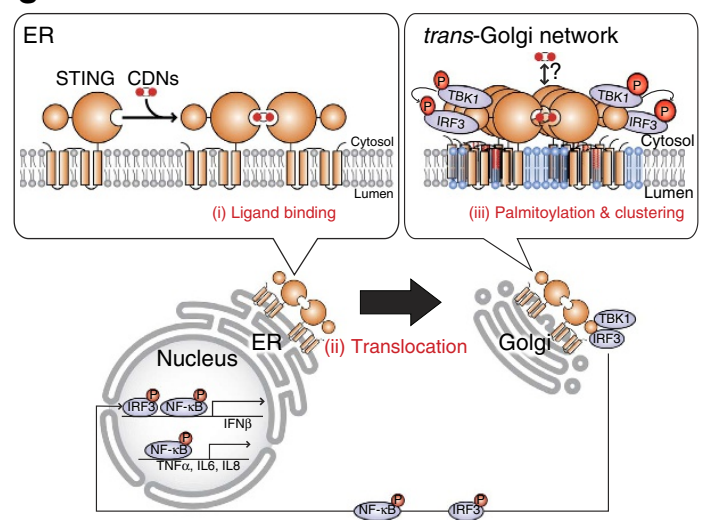

Figure 5 | Disturbing Golgi lipid order suppresses the STING-dependent type I interferon response. (a) emsCOS-1 cells were treated with $20 \mu \mathrm{M}$ D-ceramide-C6 ( $\mathrm{N}$-hexanoyl-D-erythro-sphingosine) or its non-metabolizable enantiomer L-ceramide-C6 ( $\mathrm{N}$-hexanoyl-L-erythro-sphingosine) for $1 \mathrm{~h}$. Cells were stimulated with DMXAA for $1 \mathrm{~h}$. Cell lysates were then prepared and analysed by western blot. (b-d) primary MEFs were treated with $20 \mu \mathrm{M}$ D-ceramide-C6 or L-ceramide-C6 for $1 \mathrm{~h}$. Cells were stimulated with ISD (b,c) or cGAMP (d) using Lipofectamine 2000 for $3 \mathrm{~h}$. qRT-PCR of the expression of IFN $\beta(\mathbf{b}, \mathbf{d})$ and Ifit2 (c) was performed. (e) emsCOS-1 cells processed as indicated were fixed, permeabilized and stained for GM130 (red). Nuclei were stained with DAPI (blue). Scale bars, $10 \mu \mathrm{m}$. (f) The effect of $20 \mu \mathrm{M}$ D-ceramide-C6 on the palmitoylation of STING in emsCOS-1 cells. (g) a proposed mechanism of STING activation. (i) CDNs bind to STING at the ER, (ii) STING then translocates from the ER to the Golgi. (iii) STING is palmitoylated at the Golgi, and with the aid of the lipid rafts (light blue) present in the TGN, palmitoylated STING is clustered, which facilitates the STING signalling through the recruitment of TBK1 and IRF3. ${ }^{\star} P<0.01,{ }^{\star \star} P<0.005,{ }^{\star \star \star} P P<0.001, N S$, not significant (one-way analysis of variance). 
interferon response ${ }^{12}$. With this method, we confirmed that human STING (WT) could activate IRF3-, IFN $\beta$ - or NF- $\mathrm{B}$ promoters (Supplementary Fig. 13f-h), induce IFNB and IFIT1 (Supplementary Fig. 13i-j), and promote phosphorylation of IRF3 following cGAMP stimulation (Supplementary Fig. 13k). In contrast, human STING (C88/91S) could not activate these same type I interferon responses (Supplementary Fig. 13f-k), suggesting that palmitoylation was also essential for cGAMP-induced STING activation.

The C88/91S mutant was used to determine whether palmitoylation is also needed in the host defense response against DNA viruses ${ }^{6}$. Reconstituting Sting ${ }^{-/-}$MEFs with human STING (WT) resulted in the induction of Ifnb and Il6, and phosphorylation of TBK1, IRF3 and p65 $(\mathrm{NF}-\kappa \mathrm{B})$ on infection with herpes simplex virus-1 (HSV-1), a double-stranded DNA virus (Fig. 3a-c), whereas reconstituting the MEFs with human STING (C88/91S) failed to activate the type I interferon response. STING (C88/91S) was also unable to stimulate the type I interferon response following infection with HSV-1 $\gamma 34.5$, a HSV-1 variant (Fig. 3d-f). Significantly, human STING (WT) suppressed HSV-1 replication, whereas human STING (C88/91S) could not (Fig. $3 \mathrm{~g}$ ), suggesting that the palmitoylation of STING is essential for virus resistance.

SAVI-STINGs require palmitoylation for their activity. In 2014, mutations in human STING were found in patients with early-onset systemic inflammation, cutaneous vasculopathy and pulmonary inflammation ${ }^{19,20}$. The disease was named SAVI. SAVI patients have a point mutation in exon 5 of STING (V147L, N154S or V155M). Such STING variants exhibit a gainof-function phenotype and are able to stimulate the production of an IFN $\beta$ reporter construct irrespective of the presence of cGAMP $^{19,20}$. When transiently expressed in HEK293T cells, all the SAVI-STINGs localized to perinuclear compartments, but not to the ER (Fig. 4a), which is consistent with previous observations ${ }^{20,21}$. We further found that some portion of SAVI-STINGs localized to the TGN (Fig. 4b), where STING was activated (Fig. 1e-h). We then examined whether the palmitoylation was also required for the activity of the SAVI-STINGs. The three SAVI-STINGs (V147L, N154S and V155M), when transiently expressed in HEK293T cells, resulted in activation of the IRF3-, IFN $\beta$ - and NF- $\kappa B$-promoters (Fig. 4c-e), and the phosphorylation of IRF3 (Fig. 4f). In contrast, SAVI-STINGs with the C88/91S mutation could not activate the IRF3-, IFN $\beta$ - or NF- $\mathrm{BB}$-promoters (Fig. $4 \mathrm{c}-\mathrm{e}$ ), and could not induce the phosphorylation of IRF3 (Fig. 4f). We also examined effects of 2-BP on the SAVI-STING-induced type I interferon response. HEK293T cells that stably express SAVI-STING (V147L and N154S) showed the type I interferon responses under unstimulated conditions. Treatment of the cells with 2-BP significantly reduced the type I interferon responses, such as induction of the IRF3 promoter (Fig. $4 \mathrm{~g}$ ) and phosphorylation of IRF3 (Fig. 4h). Thus, inhibition of palmitoylation could suppress a gain-of-function phenotype in the SAVI-STING. Effects of 2-BP was not determined on V155M variant, because stable cell lines could not be generated.

Disturbing Golgi lipid order suppresses STING activation. Protein palmitoylation has been implicated in the clustering of a number of proteins ${ }^{30}$ such as $\mathrm{H}$-ras and Fas into lipid rafts (specific membrane domains enriched in cholesterol and sphingomyelin (SM)). Clustering of STING is proposed to bring TBK1 and IRF3 into close proximity, so that TBK1 can phosphorylate IRF3 (ref. 33). Palmitoylation of STING may facilitate the clustering of STING into lipid rafts at the TGN.
Cholesterol is suggested to be enriched at the TGN, and cholesterol together with SM generated by SM synthase 1 are thought to form lipid rafts at the $\mathrm{TGN}^{34}$. Treatment of cells with D-ceramide-C6 disrupts lipid rafts at the Golgi by generating short-chain SM that disturbs the lipid $\operatorname{order}^{34}$. Of note, D-ceramide-C6 inhibited the STING-dependent phosphorylation of TBK1 and IRF3 (Fig. 5a) and the induction of Ifnb and Ifit2 (Fig. 5b-d) without affecting the translocation of STING to the Golgi (Fig. 5e) or the palmitoylation of STING (Fig. 5f). D-ceramide-C6 had only marginal effects on the RLR pathway (Supplementary Fig. 14). L-ceramide-C6, a non-metabolizable enantiomer of D-ceramide-C6, did not inhibit the phosphorylation of TBK1 and IRF3. We propose that palmitoylation allows clustering of STING at the lipid rafts of the TGN, which facilitates the type I interferon response by bringing TBK1 and IRF3 closer to each other (Fig. 5g).

Proteins containing DHHC cysteine-rich domains (DHHC proteins) are protein palmitoyltransferases. The human genome encodes 23 DHHC proteins ${ }^{35}$. By overexpression of individual DHHC proteins in emsCOS-1 cells followed by metabolic labelling with $\left[{ }^{3} \mathrm{H}\right]$ palmitate, we found that DHHC3, DHHC7 and DHHC15 each increased the $\left[{ }^{3} \mathrm{H}\right]$ labelling of STING on stimulation (Supplementary Fig. 15a), and that this increase was dependent on their enzymatic activities (Supplementary Fig. 15b). This result may indicate that not all of the total STING was palmitoylated on stimulation, at least in COS-1 cells. DHHC3, DHHC7 and DHHC15 all localized to the Golgi (Supplementary Fig. 15c), suggesting that these DHHC proteins may contribute to the palmitoylation of C88/91 of STING at the Golgi. Since the mobility was not different between STING (WT) and STING (C88/91S) in SDS-PAGE, we could not determine how much of STING was palmitoylated.

STING, in addition to its association with SAVI, has also been associated with inflammations that are caused by dysregulated degradation of self-DNA ${ }^{36}$. Mice lacking DNase II or DNase III (Trex1) die during embryonic development or within 10 weeks of birth, respectively, with inflammation-related phenotypes. The lethality is completely rescued by the loss of STING ${ }^{14-16}$. Mutations of human DNase III are found in inflammatory disorders such as Aicardi-Goutieres syndrome and systemic lupus erythematosus ${ }^{18}$. Leakage of mitochondrial DNA into the cytoplasm, which occurs in many human diseases and ageing, induces STING-dependent inflammatory responses ${ }^{37}$. Our findings offer new opportunities to treat such cytosolic DNA-triggered inflammatory diseases by suppressing the palmitoylation of STING.

\section{Methods}

Antibodies. For the immunoprecipitation of endogenous STING, the rabbit polyclonal anti-STING antibody was used ${ }^{23}$ (dilution 1:100 for immunoprecipitation and dilution 1:50 for immunofluorescence). Other antibodies used in this study were as follows: mouse anti-GFP (JL-8, dilution 1:1,000; Clontech); mouse anti-GFP (3E6, dilution 1:500), Alexa 488-, 594- or 647conjugated secondary antibodies (A21202, A21203, A21206, A21207, A31573, A11016, A21448, dilution 1:2,000; Life Technologies); rabbit anti-TBK1 (ab40676, dilution 1:1,000; Abcam); rabbit anti-phospho-TBK1 (D52C2, dilution 1:1,000 for western blotting, dilution 1:100 for immunofluorescence, dilution 1:50 for immunoelectron microscopy), rabbit anti-phospho-IRF3 (4D4G, dilution 1:1,000) rabbit anti-p65 (D14E12, dilution 1:1,000), and rabbit anti-phospho-p65 (93H1, dilution 1:1,000; Cell Signalling); rabbit anti-IRF3 (FL-425, dilution 1:200; Santa Cruz); rabbit anti-Rab11 (71-5300, dilution 1:100; Zymed); mouse anti-p62 (610382, dilution 1:1,000), mouse anti-calreticulin (612136, dilution 1:200) and mouse anti-GM130 (610823, dilution 1:1,000) (BD Biosciences); mouse anti- $\alpha$ tubulin (DM1A, dilution 1:5,000; Sigma); sheep anti-mouse IgG antibody-HRP (NA9310V, dilution 1:4,000) and donkey anti-rabbit IgG antibody-HRP (NA9340V, dilution 1:4,000) (GE Healthcare); sheep anti-TGN46 (AHP500G, dilution 1:4,000 for immunofluorescence, dilution 1:200 for immunoelectron microscopy) and sheep anti-TGN38 (AHR499G, dilution 1:200) (Serotec); rabbit anti-STING antibody (19851-1-AP, dilution 1:1,000 for western blotting; Proteintech); rabbit anti-syntaxin5 (110,053, dilution 1:200; Synaptic Systems); 
mouse anti-HA (4B2, dilution 1:1,000 for western blotting and immunofluorescence; Wako); donkey anti-mouse IgG $(\mathrm{H}+\mathrm{L})$ conjugated with DyLight 405 (715-475-150, dilution 1:2,000), colloidal gold (CG) particleconjugated donkey anti-rabbit antibody (12 nm; 711-205-152, dilution 1:20) and donkey anti-sheep antibody (6 nm; 713-195-147, dilution 1:20) (Jackson ImmunoResearch laboratories).

Reagents. The following reagents were purchased from the manufacturers as noted: DMXAA, BFA, hydroxylamine-HCl, poly (I:C) and nocodazole (Sigma); 2-BP (Wako); Palmitate [9,10-3H(N)] (American Radiolabeled Chemicals Inc); 2', $3^{\prime}$-cGAMP (InvivoGen); D-ceramide-C6 (Cayman); L-ceramide-C6 (Matreya Inc). ISD (90-mer), used as dsDNA in this study, was prepared as follows ${ }^{7,38}$ : equimolar amounts of oligonucleotides (sense: $5^{\prime}$-TACAGATCTACTAGTGATCT ATGACTGATCTGTACATGATCTACATACAGATCTACTAGTGATCTATGAC TGATCTGTACATGATCTACA-3' ${ }^{\prime}$, antisense: 5' -TGTAGATCATGTACAGATC AGTCATAGATCACTAGTAGATCTGTATGTAGATCATGTACAGATCAGTC ATAGATCACTAGTAGATCTGTA- $3^{\prime}$ ) were annealed in PBS at $70^{\circ} \mathrm{C}$ for $30 \mathrm{~min}$ before cooling to room temperature.

Cell culture. COS-1 and HEK293T cells were purchased from the American Type Culture Collection (ATCC). Primary MEFs were obtained from embryos of WT or Sting ${ }^{-/-}$mice at E13.5. BMDMs were differentiated from bone marrow cells using L929-conditioned medium ${ }^{7}$. COS-1, HEK293T and MEFs were cultured in DMEM supplemented with $10 \%$ foetal bovine serum/penicillin/streptomycin/ glutamine in a $5 \% \mathrm{CO}_{2}$ incubator. BMDMs were cultured in RPMI supplemented with $10 \%$ foetal bovine serum/penicillin/streptomycin/glutamine.

Reconstituted primary Sting ${ }^{-/-}$MEFs with human STINGs were obtained using retrovirus ${ }^{23}$. Plat-E cells were transfected with pBabe-puro-STING and the medium that contains the retrovirus was collected. Sting ${ }^{-/-}$MEFs were incubated with the medium and then selected with puromycin for a week.

emsCOS-1 cells were established using retrovirus transfection: HEK293T cells were transfected with pMXs-IP-EGFP-mSTING together with pCG-VSV-G and pCG-gag-pol, and the medium that contains the retrovirus was collected. COS-1 cells were incubated with the medium and then selected with puromycin for a week.

HEK293T cells that stably express human STINGs (WT, V147L or N154S) were established using retrovirus: Plat-E cells were transfected with pBabe-puro-STING together with pCG-VSV-G and the medium that contains the retrovirus was collected. HEK293T cells were incubated with the medium and then selected with puromycin for a week.

PCR cloning. Mouse STING was amplified by PCR with complementary DNA (cDNA) derived from ICR mouse liver using the following primers: $5^{\prime}$-CCC GAATTCAATGCCATACTCCAACCTGCA-3' (mSTING; sense primer, EcoRI site is underlined) and $5^{\prime}$-CCCGCGGCCGCTCAGATGAGGTCAGTGCGGA-3' (mSTING; antisense primer, NotI site is underlined). The product encoding mSTING was introduced into pMXs-IP-GFP, to generate N-terminal GFP-tagged construct. STING Cys mutants were generated by site-directed mutagenesis.

Luciferase assay. HEK293T cells seeded on 24-well plates were transiently transfected with luciferase reporter plasmid (100 ng), pRL-TK (10 ng) as internal control and STING-expression plasmid in pBabe vector (200 ng). Twenty four hours after the transfection, the luciferase activity in the total cell lysate was measured.

cGAMP stimulation of STING was performed as follows ${ }^{39}$ : Cell medium was aspirated and replaced with $200 \mu \mathrm{l}$ of $300 \mathrm{ng} \mathrm{ml}^{-1}$ cGAMP, $50 \mathrm{mM}$ HEPES (pH 7.0), $100 \mathrm{mM} \mathrm{KCl}, 3 \mathrm{mM} \mathrm{MgCl}_{2}, 0.1 \mathrm{mM}$ DTT, $85 \mathrm{mM}$ sucrose, $0.2 \%$ BSA, $1 \mathrm{mM}$ ATP and $10 \mu \mathrm{g} \mathrm{ml}^{-1}$ digitonin. Cells were incubated for $30 \mathrm{~min}$ at $37^{\circ} \mathrm{C}$ The medium was then replaced with $500 \mu \mathrm{l}$ of fresh growth media without antibiotics. Eight hours after cGAMP stimulation, luciferase activity in the total cell lysate was measured.

qRT-PCR. Total RNA was extracted from cells using Isogen II (Nippongene), purified using High Pure RNA Tissue kit (Roche) and reverse-transcribed using the High Capacity cDNA Reverse Transcription kit (Applied Biosystems). Quantitative real-time PCR (qRT-PCR) was performed using LightCycler 480 SYBR Green I Master (Roche) and LightCycler 480 (Roche). The sequences of the primers were as follows. 5'-GACCAACAAGTGTCTCCTCCAAA-3' (human IFNB; sense primer) and $5^{\prime}$-AGCAAGTTGTAGCTCATGGAAAGAG-3' (human IFNB; antisense primer); $5^{\prime}$-AGGTGCAGTTTTGCCAAGGA-3' (human IL8; sense primer) and $5^{\prime}$-TTTCTGTGTTGGCGCAGTGT-3' (human IL 8 ; antisense primer); $5^{\prime}$-ACTTT GGAGTGATCGGCCCCCAGA-3' (human TNF; sense primer) and $5^{\prime}$-GCTTGTC ACTCGGGGTTCGAGAAGA-3' (human TNF; antisense primer); $5^{\prime}$-GCCAAGG TCATCCATGACAACT-3' (human GAPDH; sense primer) and $5^{\prime}$-GAGGGGC CATCCACAGTCTT-3' (human GAPDH; antisense primer); 5'-CAGCTCCAAG AAAGGACGAAC- $3^{\prime}$ (mouse If $n b$; sense primer) and $5^{\prime}$-GGCAGTGTAACTCTT CTGCAT- $3^{\prime}$ (mouse $I f n b$; antisense primer); $5^{\prime}$-TAGTCCTTCCTACCCCAAT TTC-3' (mouse Il6; sense primer) and 5'-TTGGTCCTTAGCCACTCCTTC-3' (mouse Il6; antisense primer); 5'-AGGTCGGTGTGAACGGATTTG-3' (mouse Gapdh; sense primer) and 5'-TGTAGACCATGTAGTTGAGGTCA-3' (mouse $G a p d h$; antisense primer). Target gene expression was normalized on the basis of GAPDH content.

Immunocytochemistry. Cells were fixed with $4 \%$ paraformaldehyde (PFA) in PBS at room temperature for $15 \mathrm{~min}$, permeabilized with $0.1 \%$ Triton X-100 in PBS at room temperature for $5 \mathrm{~min}$ and quenched with $50 \mathrm{mM} \mathrm{NH}_{4} \mathrm{Cl}$ in $\mathrm{PBS}$ at room temperature for $10 \mathrm{~min}$. After blocking with $3 \%$ BSA in PBS, cells were incubated with primary antibodies, then with secondary antibodies conjugated with Alexa fluorophore.

Confocal microscopy. Confocal microscopy was performed using a TCS SP8 (Leica) with a $63 \times 1.2$ Plan-Apochromat water immersion lens.

Nocodazole treatment. Cells were treated with nocodazole $(20 \mu \mathrm{M})$ for $30 \mathrm{~min}$, stimulated with DMXAA $\left(25 \mu \mathrm{g} \mathrm{ml}^{-1}\right)$ in the presence of nocodazole for $100 \mathrm{~min}$, then fixed and stained.

Immunoelectron microscopy. emsCOS-1 cells were stimulated with DMXAA for $1 \mathrm{~h}$ and fixed with 4\% PFA (1.04005.1000, MERCK), 4\% sucrose and $0.1 \mathrm{M}$ phosphate buffer ( $\mathrm{pH} 7.2$ ) for $10 \mathrm{~min}$ at room temperature and then $30 \mathrm{~min}$ at $4{ }^{\circ} \mathrm{C}$. After rinsing with $7.5 \%$ sucrose and $0.1 \mathrm{M}$ phosphate buffer ( $\mathrm{pH} 7.4$ ), the cells were scraped and embedded in $10 \%$ gelatin (G2500, Sigma) and $0.1 \mathrm{M}$ phosphate buffer ( $\mathrm{pH}$ 7.4). The cell blocks were cut into small pieces (about $1 \mathrm{~mm}$ cube), which were infused overnight with $20 \%$ polyvinylpyrrolidone (PVP10, Sigma-Aldrich), $1.84 \mathrm{M}$ sucrose, $10 \mathrm{mM} \mathrm{Na}_{2} \mathrm{CO}_{3}$ and $0.08 \mathrm{M}$ phosphate buffer ( $\mathrm{pH} 7.4$ ) followed by rapid freezing in liquid nitrogen ${ }^{40}$. Ultrathin cryosections were prepared using an ultramicrotome (EM UC7, Leica) equipped with a cryochamber (EM FC7, Leica). They were incubated with $1 \%$ BSA and PBS for $20 \mathrm{~min}$ at room temperature, and then with two primary antibodies, anti-p-TBK1 (1:50, D52C2) and anti-TGN46 (1:200, AHP500GT, Bio-Rad), for 3 days at $4{ }^{\circ} \mathrm{C}$. After incubation with two CG-conjugated secondary antibodies, $6 \mathrm{~nm}$ CG-donkey anti-sheep (1:20, 713-195-147) and 12 nm CG-donkey anti-rabbit (1:20, 711-205-152, Jackson ImmunoResearch), for $1 \mathrm{~h}$ at room temperature, they were fixed with $2 \%$ glutaraldehyde (G017/1, TAAB) and PBS for $5 \mathrm{~min}$. They were stained with $2 \%$ uranyl acetate for 5 min and embedded in $0.17 \%$ uranyl acetate and $0.33 \%$ polyvinyl alcohol (P8136, Sigma-Aldrich). After drying up, sections were observed using an electron microscope (JEM1200EX, JEOL).

Western blotting. Proteins were separated in polyacrylamide gel and then transferred to polyvinylidene difluoride membranes (Millipore). These membranes were incubated with primary antibodies, followed by secondary antibodies conjugated to peroxidase. The proteins were visualized by enhanced chemiluminescence using a LAS-4000 (GE Healthcare). Uncropped images are shown in Supplementary Fig. 16

Metabolic labelling with $\left[^{3} \mathbf{H}\right]$ palmitate. emsCOS- 1 cells were incubated in DMEM containing $0.1 \%$ essentially fatty acid-free BSA for $1 \mathrm{~h}$ at $37^{\circ} \mathrm{C}$, and then metabolically labelled with $0.1 \mathrm{mCi} \mathrm{ml}^{-1}\left[{ }^{3} \mathrm{H}\right]$ palmitate at $37^{\circ} \mathrm{C}$. One hour after the labelling, DMXAA (final $25 \mu \mathrm{g} \mathrm{ml}^{-1}$ ) was added to the cell medium. After incubation for appropriate times, cells were washed with ice-cold PBS, scraped in immunoprecipitation buffer composed of $50 \mathrm{mM}$ HEPES-NaOH ( $\mathrm{pH} 7.2), 150 \mathrm{mM}$ $\mathrm{NaCl}, 5 \mathrm{mM}$ EDTA, $1 \%$ SDS, $1 \%$ Triton X-100, protease inhibitors (1 mM PMSF, $10 \mu \mathrm{g} \mathrm{ml}^{-1}$ leupeptin, $10 \mu \mathrm{g} \mathrm{ml}^{-1}$ pepstatin, $10 \mu \mathrm{g} \mathrm{ml}^{-1}$ aprotinin) and phosphatase inhibitors ( $8 \mathrm{mM} \mathrm{NaF}, 12 \mathrm{mM}$ beta-glycerophosphate, $1 \mathrm{mM} \mathrm{Na}_{3} \mathrm{VO}_{4}$, $1.2 \mathrm{mM} \mathrm{Na}_{2} \mathrm{MoO}_{4}, 5 \mu \mathrm{M}$ cantharidin and $2 \mathrm{mM}$ imidazole). The lysates were then sonicated on ice and diluted to $0.1 \%$ SDS. After centrifugation at 15,000 r.p.m. for $10 \mathrm{~min}$ at $4{ }^{\circ} \mathrm{C}$, the resultant supernatants were incubated for overnight at $4{ }^{\circ} \mathrm{C}$ with anti-GFP (3E6), and then incubated for $3 \mathrm{~h}$ with protein $\mathrm{G}$ Sepharose fast flow (GE Healthcare). The beads were washed four times with immunoprecipitation wash buffer (50 mM HEPES-NaOH (pH 7.2), $150 \mathrm{mM} \mathrm{NaCl,} 0.1 \%$ Triton X-100) and eluted with $2 \times$ Laemmli sample Buffer. The immunoprecipitated proteins were separated with SDS-PAGE and transferred to PVDF membrane, then autoradiographed with BAS-IP TR2040 and Typhoon9000 (GE Healthcare). In the case of MEF cells, immunoprecipitation buffer without SDS and anti-STING polyclonal antibody were used. For hydroxylamine treatment ${ }^{32}$, PVDF membranes were soaked in $1 \mathrm{M}$ hydroxylamine ( $\mathrm{pH} 7.0) / 1 \% \mathrm{SDS}$ for $3 \mathrm{~h}$ at room temperature.

Virus infection. HSV-1 (KOS strain) was purchased from ATCC. HSV-1 g34.5 was kindly provided by Dr Bernard Roizman (The University of Chicago).

Statistical analyses. Error bars displayed throughout this study represent s.e.m. unless otherwise indicated, and were calculated from triplicate or quadruplicate samples. Statistical significance was determined with one-way analysis of variance followed by Tukey-Kramer post hoc test.; ${ }^{\star} P<0.05$; ${ }^{* *} P<0.01$; ${ }^{* *} P<0.001$; 
NS, not significant $(P>0.05)$. Data shown are representative of two to three independent experiments, including microscopy images and western blots.

Data availability. The data that support the findings of this study are available from the corresponding author upon request.

\section{References}

1. Palm, N. W. \& Medzhitov, R. Pattern recognition receptors and control of adaptive immunity. Immunol. Rev. 227, 221-233 (2009).

2. Takeuchi, O. \& Akira, S. Pattern recognition receptors and inflammation. Cell 140, 805-820 (2010).

3. Blasius, A. L. \& Beutler, B. Intracellular toll-like receptors. Immunity 32, 305-315 (2010).

4. Yoneyama, M. et al. The RNA helicase RIG-I has an essential function in double-stranded RNA-induced innate antiviral responses. Nat. Immunol. 5, 730-737 (2004)

5. Ye, Z. \& Ting, J. P. NLR, the nucleotide-binding domain leucine-rich repeat containing gene family. Curr. Opin. Immunol. 20, 3-9 (2008).

6. Ishikawa, H., Ma, Z. \& Barber, G. N. STING regulates intracellular DNA-mediated, type I interferon-dependent innate immunity. Nature 461, 788-792 (2009)

7. Ishikawa, H. \& Barber, G. N. STING is an endoplasmic reticulum adaptor that facilitates innate immune signalling. Nature 455, 674-678 (2008).

8. Zhong, B. et al. The adaptor protein MITA links virus-sensing receptors to IRF3 transcription factor activation. Immunity 29, 538-550 (2008).

9. Sun, W. et al. ERIS, an endoplasmic reticulum IFN stimulator, activates innate immune signalling through dimerization. Proc. Natl Acad. Sci. USA 106, 8653-8658 (2009).

10. Jin, L. et al. MPYS, a novel membrane tetraspanner, is associated with major histocompatibility complex class II and mediates transduction of apoptotic signals. Mol. Cell. Biol. 28, 5014-5026 (2008).

11. Wu, J. et al. Cyclic GMP-AMP is an endogenous second messenger in innate immune signalling by cytosolic DNA. Science 339, 826-830 (2013).

12. Burdette, D. L. et al. STING is a direct innate immune sensor of cyclic di-GMP. Nature 478, 515-518 (2011).

13. Abe, T. et al. STING recognition of cytoplasmic DNA instigates cellular defense. Mol. Cell 50, 5-15 (2013).

14. Gall, A. et al. Autoimmunity initiates in nonhematopoietic cells and progresses via lymphocytes in an interferon-dependent autoimmune disease. Immunity 36, 120-131 (2012).

15. Ahn, J., Gutman, D., Saijo, S. \& Barber, G. N. STING manifests self DNA-dependent inflammatory disease. Proc. Natl Acad. Sci. USA 109, 19386-19391 (2012)

16. Ahn, J., Ruiz, P. \& Barber, G. N. Intrinsic self-DNA triggers inflammatory disease dependent on STING. J. Immunol. 193, 4634-4642 (2014).

17. Lee-Kirsch, M. A. et al. Mutations in the gene encoding the 3'-5' DNA exonuclease TREX1 are associated with systemic lupus erythematosus. Nat. Genet. 39, 1065-1067 (2007).

18. Rice, G. et al. Heterozygous mutations in TREX1 cause familial chilblain lupus and dominant Aicardi-Goutieres syndrome. Am. J. Hum. Genet. 80, 811-815 (2007).

19. Liu, Y. et al. Activated STING in a vascular and pulmonary syndrome. N. Engl. J. Med. 371, 507-518 (2014).

20. Jeremiah, N. et al. Inherited STING-activating mutation underlies a familial inflammatory syndrome with lupus-like manifestations. J. Clin. Invest. 124, 5516-5520 (2014).

21. Dobbs, N. et al. STING activation by translocation from the ER is associated with infection and autoinflammatory disease. Cell Host Microbe 18, 157-168 (2015).

22. Saitoh, T. et al. Atg9a controls dsDNA-driven dynamic translocation of STING and the innate immune response. Proc. Natl Acad. Sci. USA 106, 20842-20846 (2009).

23. Konno, H., Konno, K. \& Barber, G. N. Cyclic dinucleotides trigger ULK1 (ATG1) phosphorylation of STING to prevent sustained innate immune signalling. Cell 155, 688-698 (2013).

24. Misaki, R., Nakagawa, T., Fukuda, M., Taniguchi, N. \& Taguchi, T. Spatial segregation of degradation- and recycling-trafficking pathways in COS-1 cells. Biochem. Biophys. Res. Commun. 360, 580-585 (2007).

25. Soulat, D. et al. The DEAD-box helicase DDX3X is a critical component of the TANK-binding kinase 1-dependent innate immune response. EMBO J. 27, 2135-2146 (2008).

26. Lippincott-Schwartz, J. et al. Microtubule-dependent retrograde transport of proteins into the ER in the presence of brefeldin A suggests an ER recycling pathway. Cell 60, 821-836 (1990).
27. Griffiths, G. \& Simons, K. The trans Golgi network: sorting at the exit site of the Golgi complex. Science 234, 438-443 (1986).

28. De Matteis, M. A. \& Luini, A. Exiting the Golgi complex. Nat. Rev. Mol. Cell Biol. 9, 273-284 (2008)

29. Shima, D. T., Haldar, K., Pepperkok, R., Watson, R. \& Warren, G. Partitioning of the Golgi apparatus during mitosis in living HeLa cells. J. Cell Biol. 137, 1211-1228 (1997).

30. Linder, M. E. \& Deschenes, R. J. Palmitoylation: policing protein stability and traffic. Nat. Rev. Mol. Cell Biol. 8, 74-84 (2007).

31. Ouyang, S. et al. Structural analysis of the STING adaptor protein reveals a hydrophobic dimer interface and mode of cyclic di-GMP binding. Immunity 36, 1073-1086 (2012).

32. Drisdel, R. C., Alexander, J. K., Sayeed, A. \& Green, W. N. Assays of protein palmitoylation. Methods 40, 127-134 (2006).

33. Tanaka, Y. \& Chen, Z. J. STING specifies IRF3 phosphorylation by TBK1 in the cytosolic DNA signalling pathway. Sci. Signal 5, ra20 (2012).

34. Duran, J. M. et al. Sphingomyelin organization is required for vesicle biogenesis at the Golgi complex. EMBO J. 31, 4535-4546 (2012).

35. Fukata, M., Fukata, Y., Adesnik, H., Nicoll, R. A. \& Bredt, D. S. Identification of PSD-95 palmitoylating enzymes. Neuron 44, 987-996 (2004).

36. Ahn, J. \& Barber, G. N. Self-DNA, STING-dependent signalling and the origins of autoinflammatory disease. Curr. Opin. Immunol. 31, 121-126 (2014).

37. West, A. P. et al. Mitochondrial DNA stress primes the antiviral innate immune response. Nature 520, 553-557 (2015).

38. Stetson, D. B. \& Medzhitov, R. Recognition of cytosolic DNA activates an IRF3-dependent innate immune response. Immunity 24, 93-103 (2006).

39. Woodward, J. J., Iavarone, A. T. \& Portnoy, D. A. c-di-AMP secreted by intracellular Listeria monocytogenes activates a host type I interferon response. Science 328, 1703-1705 (2010).

40. Uemura, T. et al. A cluster of thin tubular structures mediates transformation of the endoplasmic reticulum to autophagic isolation membrane. Mol. Cell. Biol. 34, 1695-1706 (2014)

\section{Acknowledgements}

We thank K. Kanno and A. Yabashi (Fukushima Medical University) for their technica assistance in immunoelectron microscopy, M. Fukata and Y. Fukata (the National Institute for Physiological Sciences) for the plasmids for DHHC palmitoyltransferases, T. Kato, S. Akagi and F. Kiku (the University of Tokyo) for their assistance, and J. McKenzie (Lawrence Berkeley National Laboratory) for comments on the manuscript. This work was supported by the Program for Promotion of Basic and Applied Researches for Innovations in Bio-oriented Industry (H.A.), Grant-in-aid for Scientific Research (S) (Grant number 20370045 to H.A.) and for Scientific Research on Innovative Areas (T.T.) from the Japanese Ministry of Education, Culture, Sports, Science and Technology, and AMED Core Research for Evolutionary medical Science and Technology (AMED-CREST) (H.A.)

\section{Author contributions}

K.M. designed and performed the experiments, analysed the data, interpreted the results and wrote the paper; H.K. performed the experiments with HSV-1 infections; T.A. performed the experiments with SAVI-STINGs; T.U. and S.W. performed the experiments using electron microscopy; T.K. aided in experiments with ceramide-C6; G.N.B. supplied reagents, designed the experiments and interpreted the results; T.T. and H.A. designed the experiments, interpreted the results and wrote the paper.

\section{Additional information}

Supplementary Information accompanies this paper at http://www.nature.com/ naturecommunications

Competing financial interests: The authors declare no competing financial interests.

Reprints and permission information is available online at http://npg.nature.com/ reprintsandpermissions/

How to cite this article: Mukai, K. et al. Activation of STING requires palmitoylation at the Golgi. Nat. Commun. 7:11932 doi: 10.1038/ncomms11932 (2016).

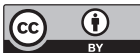

This work is licensed under a Creative Commons Attribution 4.0 International License. The images or other third party material in this article are included in the article's Creative Commons license, unless indicated otherwise in the credit line; if the material is not included under the Creative Commons license, users will need to obtain permission from the license holder to reproduce the material. To view a copy of this license, visit http://creativecommons.org/licenses/by/4.0/ 